\title{
Respuesta inicial por un grupo de Docentes Mexicanos, al cierre de escuelas por Covid-19
}

\section{Initial response of a group of Mexican teachers to school closure by Covid-19}

\author{
Emmanuel Magallanes Ulloa ${ }^{1}$ \\ Universidad Politécnica de Zacatecas (México) \\ Josué de Ávila González \\ Universidad Autónoma de Zacatecas (México)
}

Recibido: 30 de mayo de 2020

Aceptado: 21 de agosto de 2020

Publicado: 21 de septiembre de 2020

\section{Resumen}

El presente trabajo tiene por objetivo analizar el actuar de los docentes frente a la suspensión de clases presenciales debido a la contingencia sanitaria provocada por el COVID-19. Se distribuyó un cuestionario con el modelo de bola de nieve. La encuesta se realizó en fechas previas al anuncio de las estrategias nacionales y las medidas que las instituciones educativas debían tomar. Lo anterior con la finalidad de analizar las acciones realizadas por los maestros para continuar con la

${ }^{1}$ Email: emagallanes@upz.edu.mx 
actividad académica. Los resultados arrojaron que un número considerable de los docentes -en ese momento- no habían recibido capacitación para trabajar a distancia. Queda de manifiesto que las circunstancias personales de los profesores (espacios de trabajo, número de alumnos, entre otras) influyó en el hecho de trabajar en línea o no.

Palabras Clave: Covid-19; Docentes; Blended Learning; Educación. 


\section{Abstract}

This research aims to analyze the first responses of teachers to face the school closure caused by the Covid-19 health contingency. A questionnaire was distributed with the snowball model. The survey was conducted prior to the announcement of national strategies to deal with confinement measures that affected educational institutions. The above in order to analyse the actions taken by teachers to continue the activity and academic progress. The results showed that a considerable number of teachers - at that time - had not received training to work remotely. It is clear that the personal circumstances of the teachers (workspaces, number of students, among others) influenced the fact of working online or not.

Keywords: Covid-19; teacher; Blended Learning; Education. 


\section{Introducción}

La pandemia causada por el Covid-19 ha traído consigo una serie de medidas de distanciamiento y el confinamiento social que, por ende, implica el cierre de escuelas en todo México. Queda claro que esta medida es necesaria y que ante todo hay que preservar la salud de la sociedad, por otro lado, dicha acción implica una interrupción en las labores académicas, lo cual provoca un considerable atraso en la educación de miles de estudiantes de distintos niveles educativos, similar a lo ocurrido en 2009 a causa del H1N1 (Owusu-Fordjour et al., 2020).

Ante semejante dilema se buscaron estrategias para minimizar el rezago educativo que supondría la suspensión indefinida de clases. Aunque hubo algunas ideas para solucionar, está claro que la respuesta óptima se hallaba en el uso de las Tecnologías de Comunicación e Información (TICs), particularmente en las relacionadas con los Entornos Virtuales de Enseñanza y Aprendizaje (EVEAs) como un instrumento para continuar la formación, prueba de ello, es que países como Australia, Irlanda, Singapur, Alemania, Italia, entre otros, independientemente del desarrollo económico en el que se encontraran, optaron por esta opción (Crawford et al., 2020).

Es entonces que la implementación del uso de herramientas digitales pareció ser la solución ante el cierre de las escuelas, sin embargo, al estar ante una emergencia sanitaria de tal magnitud y dado el carácter imprevisible de la enfermedad y su propagación, la premura no permitió diseñar estrategias que abarcaran todos los niveles y centros educativos. Debido a la naturaleza de la contingencia no fue posible brindar capacitación, diseño de materiales, inventario de recursos tecnológicos, tanto de maestros como de estudiantes para el cambio tecnológico que demanda la situación.

Este trabajo tiene por objetivo analizar la respuesta inicial de los docentes esto es, las primeras acciones emprendidas para impartir su clase ante el cierre de las escuelas en las primeras semanas (la mayoría de las escuelas cerraron en la semana comprendida del 16 al 20 de marzo y el periodo vacacional de Semana Santa comenzó el 3 de abril) de la emergencia sanitaria. Otro objetivo es determinar las formas de trabajo en línea, así como caracterizar a los docentes según su respuesta ante la contingencia, considerando variables tales como el número de alumnos atendidos, el grado de estudio, espacio de trabajo, entre otros. Lo anterior excluye el caso de los profesores 
que carecen de conexión a internet y/o dispositivos que les permitan realizar su labor académica. El trabajo se enfoca en aquellos docentes que sí tienen acceso a la red y en la forma en que aprovecharon o no, tal herramienta.

Nos encontramos ante una emergencia sanitaria a nivel mundial, el sistema educativo en México no ha estado exento, lo cual lleva a un momento coyuntural que involucra a todos los actores; docentes, investigadores, estudiantes, padres de familia, directivos y administrativos. Ahora mismo el $60 \%$ de la población estudiantil en todo el mundo se encuentra sin clases (UNESCO, 2020). Esta coyuntura radica en el hecho de tener que dar solución a la interrogante ¿cómo continuar la formación académica de los estudiantes sin perder ciclos escolares? y por otro lado intentar abarcar los contenidos estipulados en los planes y programas previamente establecidos.

Para hacer frente a este reto planteado, la solución tecnológica es una buena carta a jugar, sin embargo, se debe considerar la brecha digital tan pronunciada que existe en México. El sistema educativo del país, los gobiernos estatales y federales han tenido la asignatura pendiente de costear el acceso a internet. Así pues, la cobertura de servicio de internet se presenta como el primer escollo para usar la tecnología como una herramienta educativa en esta contingencia:

En el caso de México esto se ha hecho evidente en años recientes, pues de acuerdo con datos de la Encuesta Nacional sobre Disponibilidad de Tecnologías de Información en Hogares (ENDUTIH de aquí en adelante) del Instituto Nacional de Estadística y Geografía (INEGI, 2016a) más de la mitad de los hogares en el país no disponen de conexión a internet (53\%); de 32,925,270 hogares sólo 45.6\% cuentan con computadora y de $122,273,473$ habitantes $73.6 \%$ son usuarios de telefonía móvil. Las cifras anteriores reflejan que existen grupos de personas con un rezago en el acceso y uso de estas tecnologías (Martínez Domínguez, 2018, p. 2)

Además de lo citado, es necesario considerar la heterogeneidad que se presenta en nuestro país:

Otra de las causas que genera desigualdad en el acceso a internet es que no todos los estados del país han regulado este aspecto, puesto que derivado de un análisis 
de cada una de las Constituciones Estatales se encontró que sólo el $40 \%$ contempla el derecho de acceder a internet, a las redes digitales, a las TIC o a la sociedad de la información. En este sentido, de los trece estados que integran el acceso a internet en su legislación, diez presentan mayor índice de cobertura a la del promedio nacional, estos son: Baja California Sur con 75\%, Sonora 71\%, Ciudad de México 67\%, Tabasco 63\%, Tamaulipas 56\%, Querétaro 54\%, Morelos 53\%, Aguascalientes 47\%, Durango 46\% y Nayarit 46\%. Por otro lado, aunque Chiapas, Veracruz y Zacatecas conciben el derecho de acceso a internet, el índice de penetración es menor a la del promedio nacional, con $13 \%, 31 \%$ y $33 \%$ respectivamente (Alcalá, 2019, p. 63)

Aunado a lo anterior, cabe la posibilidad de no poder contribuir al esfuerzo de llevar las clases en línea, en tanto que los contratos colectivos no consideran esta eventualidad en sus cláusulas. Sin duda alguna, es una situación tan excepcional que no se previó en la relación laboral entre el maestro y la institución educativa, lo cual les da pleno derecho a algunos docentes de no intervenir (Mireles, 2020). Ciertamente esto lleva a la formación de una grieta que podría no solventarse, sin embargo, en términos jurídicos es una situación que se está presentado y por ello se hace mención.

\section{Blended Learning}

Blended Learning ha tenido un "resurgimiento" de acuerdo a la demanda de ubicuidad en los cursos en nivel superior, sobre todo, aunque se ha implementado con éxito en otros niveles:

Blended learning designs have steadily increased as a favored course delivery model alongside fully online options. Previously defined by the proportions of faceto-face versus online coursework, blended learning is typified by the integration of those digital solutions most applicable for achieving the learning outcomes of the course. Media-rich digital learning platforms, personalized or adaptive courseware, and web conferencing tools capable of connecting students for synchronous distance activities are becoming common solutions for blended learning designs. Students report a preference for blended learning, citing flexibility, ease of access, and the integration of sophisticated multimedia. [Los diseños de aprendizaje mixto han aumentado constantemente como modelo preferido de entrega de cursos junto con opciones totalmente en línea. Anteriormente definido por las proporciones de los cursos presenciales frente a los cursos en línea, el aprendizaje combinado se 
caracteriza por la integración de las soluciones digitales más aplicables para lograr los resultados del aprendizaje del curso. Las plataformas de aprendizaje digital con abundancia de medios, los programas informáticos de cursos personalizados o adaptables y las herramientas de conferencias web capaces de conectar a los estudiantes para realizar actividades a distancia sincrónicas se están convirtiendo en soluciones comunes para los diseños de aprendizaje combinado. Los estudiantes informan de su preferencia por la formación combinada, citando la flexibilidad, la facilidad de acceso y la integración de multimedia sofisticada] (Alexander et al., 2019, p. 23)

Aunque hay diversas formas de definirlo y algunas variantes, Alexander y otros autores consideran el Blended Learning como una mezcla de trabajo presencial asistido por plataformas digitales ¿Un nuevo término o un nuevo concepto? Es la pregunta que detona el trabajo de los autores y es sin duda pertinente; a menudo en la ciencia se nombran conceptos o cosas, como si fueran nuevos, cuando en realidad no hay novedad alguna y con ello se provoca confusión. Volviendo al concepto, éste trata sobre la mezcla de cuatro variables: la localización, la sincronía, la distribución y la instrucción. La mezcla de dichos componentes es justamente el Blended Learnning (BL), esto es una composición entre el aprendizaje en línea, a distancia, sincrónico, asincrónico, activo, pasivo y todas las posibles variables (Bartolomé et al., 2017; Salinas Ibáñez et al., 2018). De lo anterior se destaca este concepto para el propósito de la tarea. El BL es considerado también un ambiente virtual de enseñanza, aunque con la peculiaridad de que el grado de virtualidad es diverso, en relación con los componentes antes mencionados. Esto supone que el aprendizaje puede tener lugar en un ambiente enteramente virtual y complementarse con sesiones presenciales, por ejemplo.

Para concluir, el BL se destaca sobre todo en términos de transición o de migración a los EVEA (Entornos Virtuales de Enseñanza y Aprendizaje) ya que ofrece la posibilidad de ir de a poco en el cambio. Para quien trabajaba en este tipo de modelos, sea alumno o docente, previo al cierre de las escuelas este avance es valioso, pues ya se cuenta con experiencia en el uso de las TICs y los EVEAs. Este hecho resultó relevante ya que, en el caso de los docentes que usaban este tipo de recursos, antes del cierre de escuelas, independientemente del grado de virtualidad o de cómo entrelazaban los elementos mencionados, al combinarlos con sus clases presenciales 
convertían su modelo de enseñanza en uno mixto, esto es, un modelo blended. Aun sin que necesariamente hubiera conciencia de ello.

\section{Metodología}

Este es un estudio exploratorio dado que, como se ha mencionado con antelación, éste se realizó en las primeras semanas del confinamiento, además no hay un claro precedente de una situación similar. Si bien, el H1N1 pudiera considerarse como antecedente, hay una década de cambios tecnológicos de por medio, por otro lado, aunque hay estudios sobre la adopción de tecnología para la enseñanza, el tiempo limitado para esto es un factor distintivo, pues como se ha señalado se trata de una primera respuesta de los profesores. El estudio también es descriptivo pues sólo se señala la presencia y características de dichas respuestas iniciales (Veiga et al., 2008). Por otra parte, se considera no experimental en tanto que el modelo bola de nieve no permite tener un control sobre la muestra. Se optó por este medio para respetar las medidas de confinamiento y sana distancia de los informantes.

A través de un cuestionario en línea se realizó la encuesta a profesores $(\mathrm{N}=204)$ procedentes de 20 estados de la República Mexicana y de diferentes niveles educativos. El cuestionario se distribuyó bajo el modelo de bola de nieve, que consiste en distribuir la encuesta por medios digítales y pidiendo al receptor que lo comparta con sus colegas, el principal motivo por el cual se eligió este modelo fue el confinamiento ocasionado por la pandemia. Otra ventaja del modelo es que requiere poca planeación y el costo económico es prácticamente nulo. Permitió llegar a un número considerable de profesores, aunque este número no se supo hasta el final, lo cual es una limitante de este modelo. Otro punto a considerar es el posible sesgo que puede darse, ya que se tiende a compartir con personas que tienen características similares, también puede llegar a poner en duda la representatividad de algunas particularidades de los encuestados.

La encuesta se realizó durante los días 17, 18, 19 de abril, cuando aún las autoridades educativas no habían precisado instrucciones acerca del actuar frente a la contingencia. Este lapso fue considerado debido a que el calendario escolar marcaba el lunes 20 de abril para el regreso a clases después de las vacaciones de Semana Santa, los docentes aún no recibían indicaciones oficiales, por lo tanto, resultó interesante conocer las acciones que se estaban tomando por iniciativa personal y/o por decisiones dentro del respectivo centro de trabajo y las dificultades a las 
que éstos se estaban enfrentando. Al regreso, la Secretaría de Educación Pública (SEP) y diversos sistemas y subsistemas implementaron estrategias, en algunos casos obligatorias, para afrontar la situación.

El cuestionario de los docentes se estructura con las siguientes secciones:

- Identificación

- Acceso al mundo digital

- Condiciones laborales

- Capacitación

- Clases en línea

- Emociones

- Reflexiones finales

En la sección de Identificación si bien se dio un tratamiento anónimo y confidencial a las respuestas de los profesores, se consideró: el estado de procedencia, la institución en la que laboran, edad, género y años de servicio. La sección de Acceso al mundo digital se consideró como una verificación acerca del acceso físico, en términos de conexión a internet y tipos de dispositivos al alcance de los docentes. En el apartado de Condiciones laborales se preguntó, número de grupos, cantidad de alumnos y materias que estaban impartiendo.

Dentro del apartado de Capacitación se consideró si tenían una formación previa a la contingencia para trabajar a distancia y en caso de tenerla, si ésta fue por iniciativa personal o por parte de su institución educativa. En la sección de Clases en línea se preguntó si habían trabajado a distancia con sus estudiantes hasta el momento de la encuesta y en caso de haberlo hecho, si ese trabajo fue anterior a la suspensión de clases o posterior. En el apartado de Emociones se consideraron los sentimientos que habían experimentado los maestros durante el confinamiento y por último, en Reflexiones finales se pidió la opinión acerca de la calidad de las clases a distancia frente a la forma presencial y qué manera de trabajo elegirían si no estuvieran en una contingencia de esta magnitud. 


\section{Resultados}

La caracterización de la muestra de los 204 docentes respecto a género, edad y experiencia docente se observa en la Tabla 1. La mayoría son mujeres, 120 (58.8\%) y 84 hombres (41.2\%), con un promedio de edad de 41.2 años entre ambos grupos. La antigüedad general fue de 14.5 años en promedio. En la Tabla 2 se observa la distribución con relación a su grado de estudio, la mitad de la muestra tiene el grado de Maestría (52.4\%), seguido del grado de Doctor (27.4\%) y por último Licenciatura (20.1\%). La gran mayoría de la muestra (86.3\%) imparte clase en sector público (Tabla 3).

Tabla 1

Distribución de la Muestra Respecto a Género, Edad y Años de Antigüedad

\begin{tabular}{lccc}
\hline \multicolumn{1}{c}{ Característica } & Mujeres & Hombres & General \\
\hline $\begin{array}{l}\text { Promedio de Edad } \\
\text { (años) }\end{array}$ & 40.3 & 42.5 & $41.2^{\mathrm{a}}$ \\
$\begin{array}{l}\text { Años de experiencia } \\
\text { docente (años) }\end{array}$ & 13.9 & 15.2 & $14.5^{\mathrm{b}}$ \\
\hline
\end{tabular}

${ }^{\text {a }}$ Edad Máxima = 66, Mínima = 23, Desviación Estándar = 9.3

b Antigüedad Máxima 45, Mínima $=1$, Desviación Estándar $=9.5$

Tabla 2

Distribución de la Muestra Respecto a Grado de Estudio

\begin{tabular}{lccc}
\hline \multicolumn{1}{r}{ Grado } & Mujeres & Hombres & General \\
\hline Doctorado & 36 & 20 & 56 \\
Maestría & 60 & 47 & 107 \\
Licenciatura & 24 & 17 & 41 \\
Total & 120 & 84 & 204 \\
\hline
\end{tabular}


Tabla 3

Distribución de la Muestra Respecto a la Modalidad de la Escuela

\begin{tabular}{lccc}
\hline \multicolumn{1}{c}{ Modalidad } & Mujeres & Hombres & General \\
\hline Pública & 104 & 72 & 176 \\
Privada & 11 & 6 & 17 \\
Ambas & 5 & 6 & 11 \\
Total & 120 & 84 & 204 \\
\hline
\end{tabular}

Si bien se logró captar en la muestra a docentes que imparten clases desde nivel primaria hasta doctorado (Tabla 4), la mitad dan clases en nivel superior (50.5\%), seguido de 81 docentes (39.7 $\%)$ que imparten a nivel preparatoria. Se tienen 64 maestros que dan en al menos dos niveles educativos distintos. En relación con el número de estudiantes que atienden los profesores en el ciclo escolar en que se realizó el estudio (Tabla 5), el rango con más maestros es de 0-50 estudiantes con 57 (27.9\%), pero los demás rangos no están tan alejados de ese número. Atender a más de 80 estudiantes ya se considera un situación complicada para trabajar a distancia (Sánchez Mendiola et al., 2020), sin embargo, en este caso existen 101 maestros (49.5\%) encuestados que están atendiendo a un número mayor de 100 estudiantes, es decir, más de la mitad de los docentes se encuentran en una situación poco favorable para desarrollar adecuadamente su labor. Dentro de la muestra se obtuvo que el promedio de grupos atendidos por cada maestro es de 4.5. 
Tabla 4

Distribución de Docentes en Relación con el Nivel en el que Imparten Clase

\begin{tabular}{lcc}
\hline \multicolumn{1}{c}{ Nivel } & Docentes & Otro Nivel $^{\mathrm{a}}$ \\
\hline Primaria & 24 & 3 \\
Secundaria & 15 & 5 \\
Preparatoria & 81 & 18 \\
Nivel Superior & 103 & 26 \\
Maestría & 5 & 4 \\
Doctorado & 2 & 2 \\
\hline
\end{tabular}

Nota. La columna de docentes suma 230 ya que hay docentes que imparten en más de un nivel educativo.

${ }^{a}$ Son maestros que dan en el nivel mencionado y al menos en otro nivel distinto.

Tabla 5

Rango de Alumnos con los que Cuentan los Docentes en el Ciclo Escolar

\begin{tabular}{cc}
\hline Rango de Alumnos: & Cantidad de Docentes: \\
\hline $0-50$ & 57 \\
$51-100$ & 46 \\
$101-150$ & 34 \\
$151-200$ & 29 \\
201 o más & 38 \\
Total & 204 \\
\hline
\end{tabular}

Dentro del apartado de equipo con el que cuentan los docentes para realizar sus actividades académicas a distancia (Tabla 6), se obtuvo que: 201 maestros (98.5 \%) cuentan con computadora, más de la mitad (64.7\%) tiene impresora y prácticamente la mitad cuenta con tableta (47.5\%). Referente a los datos acerca de la conexión a internet, 202 docentes (99.0\%) cuentan con conexión fija desde casa y sólo 2 (1.0\%) indicaron no contar con ésta. Al realizar un trabajo a distancia siendo docentes, la funcionalidad de la conexión es primordial, es por esto que se les preguntó por la estabilidad de ésta (Tabla 7). Sólo 41 docentes (20.2\%) indicaron que su conexión nunca falla, la 
mayoría indicó que algunas veces falla $(64.9 \%)$ y unos pocos señalaron que su conectividad falla frecuentemente.

\section{Tabla 6}

Equipo con el que Cuentan los Docentes

\begin{tabular}{lc}
\hline \multicolumn{1}{c}{ Equipo: } & Cantidad de Docentes: \\
\hline Computadora & 201 \\
Laptop & 193 \\
PC de escritorio & 62 \\
Impresora & 132 \\
Tablet & 97
\end{tabular}

Nota. Algunos maestros cuentan con laptop y pc de escritorio, por lo tanto, la suma de ambos equipos da más de 201.

\section{Tabla 7}

Calidad de la Conexión a Internet

\begin{tabular}{lc}
\hline \multicolumn{1}{c}{ Percepción } & Cantidad \\
\hline Nunca Falla & 41 \\
Algunas veces falla & 131 \\
Frecuentemente falla & 30 \\
Total & 202 \\
\hline
\end{tabular}

En la Tabla 8 se observa la distribución de maestros con relación al género y si está o no trabajando a distancia. De las 120 mujeres encuestadas, 79 (65.8\%) están trabajando a distancia. Mientras que, de los 84 hombres encuestados, 50 (59.5\%) realizan trabajo en línea. Se realizó un contraste dependiendo de su grado académico y si está o no trabajando a distancia (Tabla 9). Con grado de Doctorado $(\mathrm{N}=56), 48$ docentes $(85.7 \%)$ están trabajando a distancia, 58 (54.2\%) con Maestría y $23(56.1 \%)$ con Licenciatura. 
Tabla 8

Docentes por Género Trabajando o no a Distancia

\begin{tabular}{lccc}
\hline Trabaja a Distancia & Mujer & Hombre & General \\
\hline Sí & 79 & 50 & 129 \\
No & 41 & 34 & 75 \\
Total & 120 & 84 & 204 \\
\hline
\end{tabular}

Tabla 9

Docentes por Grado Académico Trabajando o no a Distancia

\begin{tabular}{lcc}
\hline \multicolumn{1}{c}{ Grado } & Trabaja a Distancia & No Trabaja a Distancia \\
\hline Doctorado & 48 & 8 \\
Maestría & 58 & 49 \\
Licenciatura & 23 & 18 \\
Total & 129 & 75 \\
\hline
\end{tabular}

En la Tabla 10 se hace una distinción entre maestros de escuelas públicas, privadas y aquellos que trabajan en las dos modalidades, se encontró que: de los maestros de escuela pública $(\mathrm{N}=176), 84$ (47.7\%) trabajaron a distancia antes de la suspensión académica, 24 (13.6\%) inició después del cierre de escuelas y 68 (38.6\%) no estaban trabajando en esta modalidad. Respecto a los docentes que imparten clases en escuelas privadas $(\mathrm{N}=17), 3$ docentes $(17.6 \%)$ tenían un trabajo a distancia previo a la suspensión, 9 (52.9\%) iniciaron después y 5 (29.4\%) no lo habían hecho. De los 108 maestros de escuela pública que trabajan a distancia al momento de la encuesta, 24 (22.2\%) comenzaron a trabajar en línea después de la suspensión de clases (Figura 1), dicho de otra manera: 2 de cada 10 iniciaron después. Por otro lado, en el sector privado, de un total de 12 docentes que estaban trabajando a distancia, 9 (75\%) iniciaron posterior a la suspensión, esto es: 8 de cada 10 comenzaron a trabajar después del cierre de escuelas (Figura 2). 


\section{Tabla 10}

Docentes que Trabajan o no a Distancia y la Modalidad de su Escuela

\begin{tabular}{lccc}
\hline \multicolumn{1}{c}{ Trabaja a Distancia } & Pública & Privada & Pública y Privada \\
\hline Sí (inició antes) $^{\mathrm{a}}$ & 84 & 3 & 7 \\
Sí (inició después) $^{\mathrm{b}}$ & 24 & 9 & 2 \\
No & 68 & 5 & 2 \\
Total & 176 & 17 & 11
\end{tabular}

${ }^{a}$ Se refiere a docentes que realizaban algún trabajo a distancia con sus alumnos antes de la contingencia sanitaria.

${ }^{\mathrm{b}}$ Hace referencia a los que comenzaron a trabajar a distancia por motivo de la contingencia.

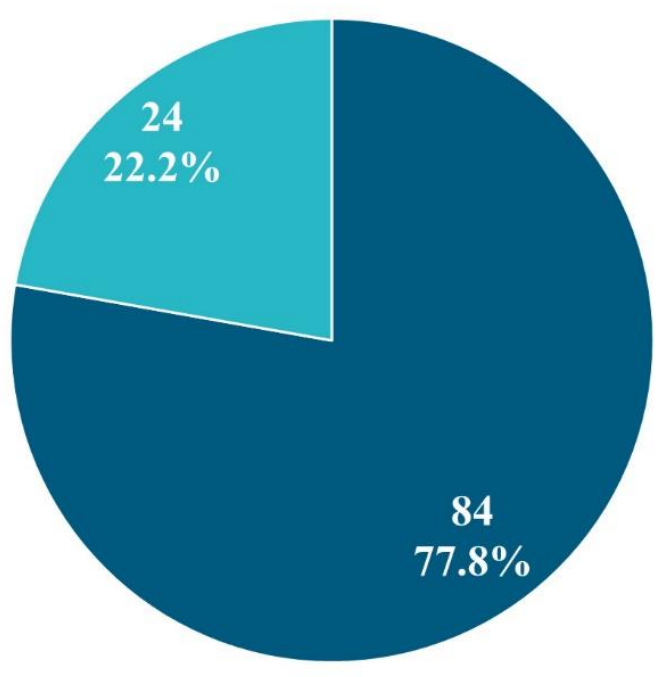

- Antes de la suspensión Después de la suspensión

Figura 1. Docentes que trabajan Blended Learning en instituciones públicas. 


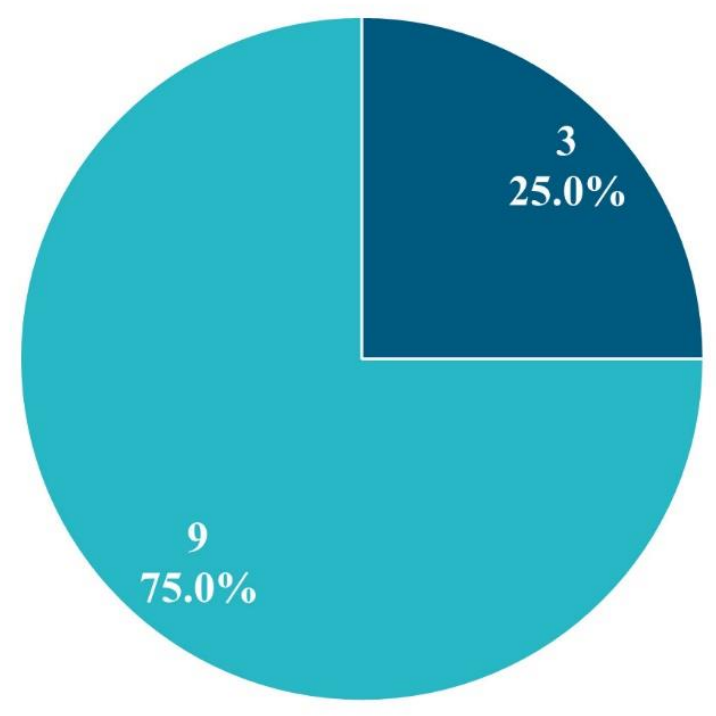

\section{Antes de la suspensión घ Después de la suspensión}

Figura 2. Docentes que trabajan Blended Learning en instituciones privadas.

Un punto importante en el presente estudio fue la búsqueda de los principales motivos por los cuales los 75 maestros no estaban realizando trabajo en línea. En la pregunta: ¿Cuál es el motivo por el que no has trabajado a distancia con tus alumnos?, dentro de la cual se podía elegir más de una opción y también estaba como posible respuesta “otra”, las cuatro respuestas más seleccionadas se observan en la Tabla 11.

Tabla 11

Motivos por los Cuales los Docentes no están Trabajando a Distancia.

\begin{tabular}{clc}
\hline Grupo & \multicolumn{1}{c}{ Respuesta } & Veces Seleccionada \\
\hline 1 & La materia no se presta & 26 \\
2 & $\begin{array}{l}\text { Falta de recursos (internet, } \\
\text { computadora) }\end{array}$ & 19 \\
3 & Falta de conocimientos tecnológicos & 19 \\
4 & Falta de tiempo & 8 \\
\hline
\end{tabular}


Para profundizar en los motivos por los cuales no estaban trabajando a distancia, se hizo una correlación entre la pregunta ¿cuál es el motivo por el que no has trabajado a distancia con tus alumnos? (Tabla 11) y la pregunta abierta: ¿cuál es el principal obstáculo que tendrías para trabajar a distancia con tus alumnos en un futuro?, obteniendo los siguientes resultados:

Grupo 1: La materia no se presta (26 docentes). Las respuestas obtenidas de la pregunta de obstáculos futuros, se pudieron clasificar en dos subgrupos: aquellos que respondieron que su materia es práctica, con un total de 10 docentes (38.5\%), algunas respuestas fueron:

- "La materia, que es práctica clínica, tal vez en parte pueda ser en línea, lo considerado de teoría dentro de la práctica”

- "No se puede dar atención a pacientes"

- "Imparto una asignatura meramente práctica”

En el segundo subgrupo: 14 maestros $(53.8 \%)$ expresan que el principal obstáculo para trabajar a distancia en un futuro es la falta de conexión y/o de equipo por parte de los estudiantes, ya que también seleccionaron la falta de recursos físicos como un impedimento para llevar a cabo dicho trabajo:

- "Que ellos no tengan la tecnología para estar trabajando a distancia, hay alumnos que no tienen computadora, mucho menos internet."

- "No todos mis alumnos tienen acceso a Internet o una computadora para acceder a Internet”

- "El acceso de los alumnos a una computadora e internet”.

Grupo 2: Falta de recursos: internet, computadora (19 docentes). En la pregunta de obstáculos futuros, de 19 maestros, las respuestas de 16 (84.2\%) de éstos se relacionan con falta de recursos por parte de sus estudiantes:

- "Muchos de mis estudiantes no cuentan con Internet o una computadora para trabajar"

- "No todos los alumnos cuentan con equipos y conectividad" 
- "De 30 alumnos, 22 de ellos no tienen las condiciones para trabajar en línea, no tienen computadora y menos internet, ni siquiera tienen smart phone”

La última respuesta llama la atención, ya que el docente comprobó el acceso que tienen sus estudiantes, de los cuales solamente 8 (26.6\%) tienen las herramientas para realizar trabajo desde casa. Las respuestas de los 3 docentes restantes tienen relación a la administración de tiempo y falta de capacitación para el uso de alguna plataforma que les permita trabajar a distancia.

Grupo 3: Falta de conocimientos tecnológicos (19 docentes). Dentro de las respuestas de estos docentes a la pregunta de obstáculos futuros, $8(42.1 \%)$ hacen referencia a la falta de acceso y/o equipo por parte de sus estudiantes, 4 maestros $(21.1 \%)$ a la falta de conocimientos tecnológicos, no aclaran específicamente si de su parte o de los alumnos, pero cabe señalar que los 4 maestros no recibieron ningún tipo de capacitación para trabajar a distancia. Las respuestas de los 7 docentes restantes hablan de cuestiones pedagógicas, problemas con plataformas, entre otras:

- "No tengo la certeza que los alumnos pongan atención"

- "Se les dificulta la comprensión, incluso en la modalidad presencial, de ahí que sea complicado hacerlo a distancia."

- "Las plataformas gratuitas son muy inestables"

Grupo 4: Falta de tiempo (8 docentes). De los 8 docentes, 6 (75\%) toman como obstáculo que no todos sus estudiantes tienen acceso a internet y/o equipo en casa, ya que los 6 también eligieron como problemática la falta de recursos. Cabe destacar que de los 8 docentes que seleccionaron la falta de tiempo, 7 son mujeres (87.5\%) y solamente 1 hombre (12.5\%). En este sentido, 2 profesoras en su respuesta hacen referencia a la dificultad de empatar el rol que tienen que desempeñar en casa con su labor profesional:

- "Disponibilidad de tiempo, al ser docente y madre de 2 niños pequeños que requieren atención la mayor parte del día, se complica tener tiempo disponible, para trabajar en línea, en horario escolar."

- "Si tienes hijos pequeños y debes cumplir con un horario específico, se puede complicar. Si no hubiera contingencia y las escuelas y/o guarderías 
están disponibles, en mi caso no habría problema. Otra desventaja es el servicio de Internet, las últimas semanas ha estado muy lento, y además, no todos los alumno cuentan con Internet o computadora para ver videos, material didáctico o entregar en línea en la fecha y hora indicada, las actividades encomendadas por los docentes."

Otra pregunta a los 75 docentes que no estaban trabajando a distancia, fue si estarían dispuestos a trabajar en línea en las próximas semanas, a lo cual 62 docentes $(82.6 \%)$ respondieron que sí, 10 (13.3\%) dijeron no estar dispuestos y 3 (4\%) no respondieron. Dentro de los motivos expuestos por los 10 docentes que no están dispuestos a trabajar a distancia en un futuro: 6 (60\%) hacen referencia a falta de recursos por parte de los alumnos para realizar dicho trabajo, 3 (30\%) mencionan que imparten una materia práctica. La respuesta del docente faltante habla de su percepción sobre la problemática de realizar un trabajo como es la enseñanza a distancia.

Dentro del estudio se preguntó si se contaba con un espacio en casa destinado para realizar labores académicas, 134 maestros (65.7\%) respondieron que sí contaban con un espacio de este tipo, mientras que 70 (34.3\%) contestaron que no. Sobre si el hecho de contar con este espacio puede influir para realizar trabajos a distancia, se encontró que: 6 de cada 10 maestros que están trabajando a distancia tienen un espacio para realizar actividades académicas (Figura 3), mientras que 7 de cada 10 maestros que no trabajan a distancia (Figura 4) cuentan con dicho espacio. También se preguntó cuál era el lugar más frecuente en casa donde se realizaban labores académicas, ya que contar con un espacio para las actividades de la escuela no determina que se realicen ahí, 3 de cada 10 maestros que cuentan con sala de estudio no realizan sus actividades académicas en ese sitio. Una correlación que se encontró fue que, 7 de cada 10 docentes que realizan actividades académicas en la sala de estudio trabajan a distancia con sus estudiantes (Figura 5). 


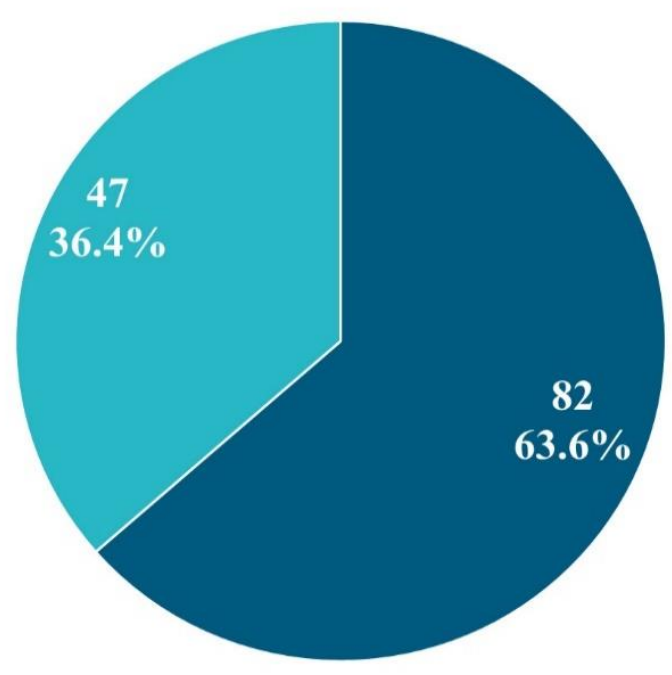

- Tiene sala de estudio $\quad$ No tiene sala de estudio

Figura 3. Docentes con o sin sala de estudio en casa que están trabajando en línea.

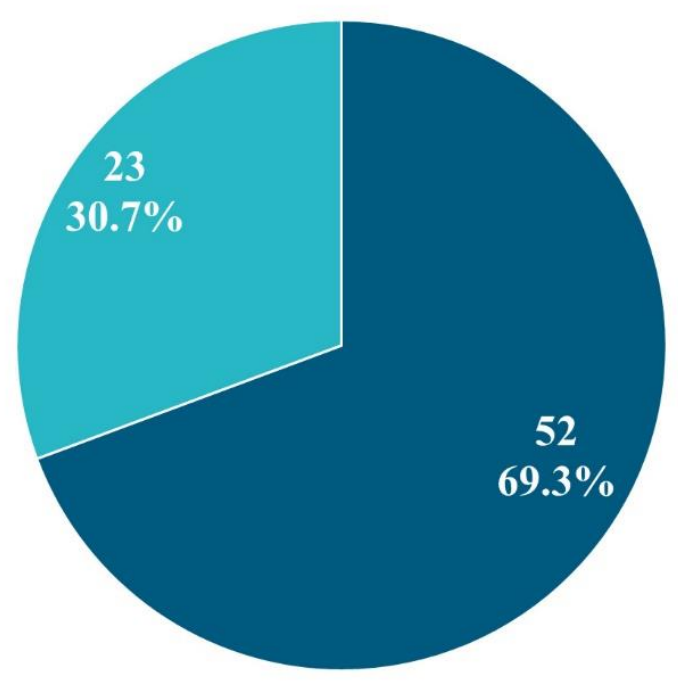

- Tiene sala de estudio $\quad$ No tiene sala de estudio

Figura 4. Docentes con o sin sala de estudio en casa que no están trabajando en línea. 


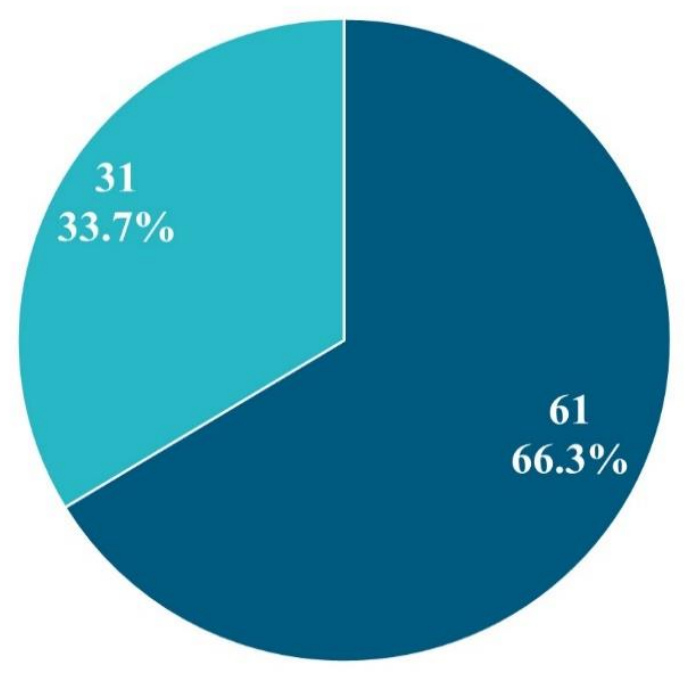

- Trabaja en línea No trabaja en línea

Figura 5. Docentes que suelen realizar sus actividades académicas en el estudio.

La Figura 6 expone que el número de alumnos asignados es una variable a considerar. Menos docentes trabajaron a distancia si el número de sus alumnos es mayor. En otros datos asociados con el trabajo en línea se destaca que 7 de cada 10 mujeres trabajan a distancia, mientras que 6 de cada 10 hombres lo hacen. El promedio de antigüedad de los que trabajan en línea es de 14.9 años y de los que no lo hacen es de 13.6 años.

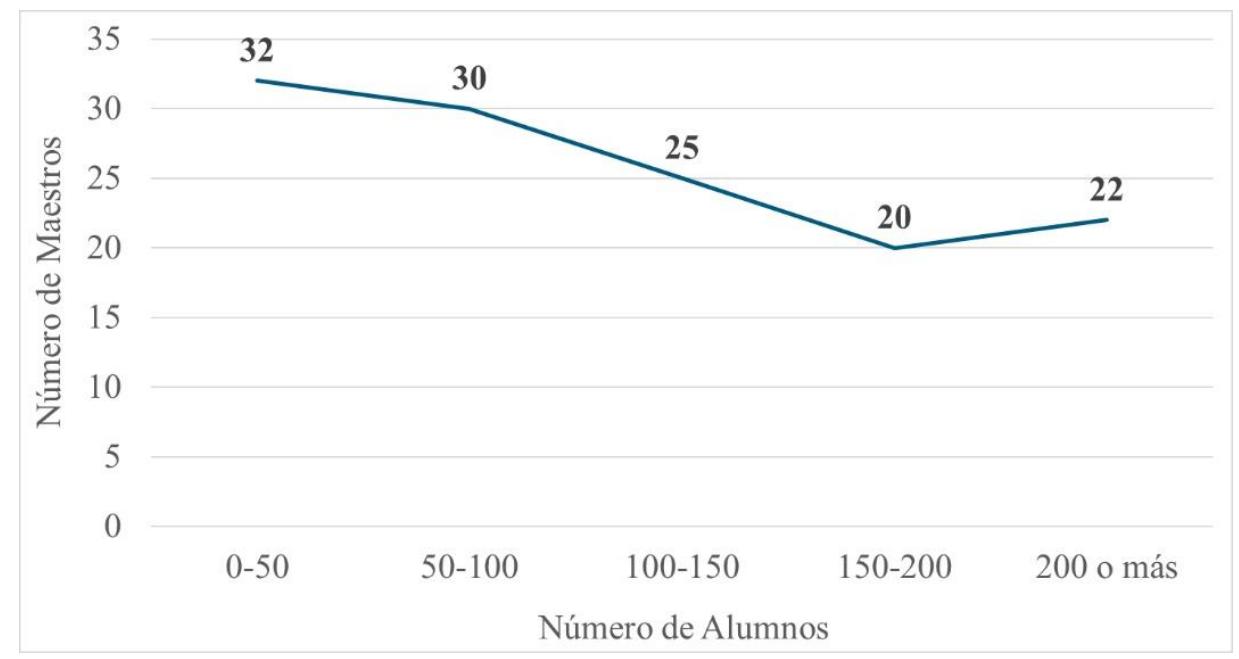

Figura 6. Docentes que trabajan distancia en contraste al número de alumnos a su cargo. 
En la búsqueda del mejor escenario para realizar un trabajo a distancia, se analizó el número de alumnos, el grado académico, el contar o no con un espacio físico en casa para desarrollar actividades académicas y la capacitación, obteniendo los siguientes resultados: de los maestros con grado de Doctor (Tabla 12) que tuvieron una capacitación para trabajar a distancia y cuentan con una sala de estudio $(\mathrm{N}=25)$, se determinó que $23(92 \%)$ trabajan a distancia. Si consideramos aquellos que tienen capacitación, sala de estudio y menos de 200 estudiantes, los 18 docentes (100\%) están trabajando en línea con sus alumnos. Mientras que los Doctores con capacitación, sala de estudio y que tienen más de 200 estudiantes $(\mathrm{N}=7), 5(71.4 \%)$ trabajan a distancia, $2(28.6 \%)$ no lo hacen. En contraste; en el escenario menos favorable, que no se tiene capacitación, ni se cuenta con una sala de estudio en casa y se tienen más de 200 alumnos, no se obtuvo ningún caso. Al considerar las variables mencionadas y tomando menos de 200 estudiantes, los 4 Doctores (100\%) están trabajando en línea. No hay registro de Doctores sin capacitación, sin espacio específico y que no estén trabajando a distancia.

Tabla 12

Contraste de características que pueden determinar el trabajo a distancia en docentes con grado de Doctorado $(N=56)$

\begin{tabular}{ccccc}
\hline Capacitación & Sala de Estudio & $\begin{array}{c}\text { Trabaja a } \\
\text { Distancia }\end{array}$ & $\begin{array}{c}\text { Menos de 200 } \\
\text { Estudiantes }\end{array}$ & $\begin{array}{c}\text { Más de 200 } \\
\text { Estudiantes }\end{array}$ \\
\hline Sí (33) & Sí (25) & Sí (23) & 18 & 5 \\
& No (2) $(8)$ & Sí (8) & 5 & 3 \\
\hline No (23) & Sí (19) & Sí (13) & & 4 \\
& No (6) & 6 & \\
& Só (4) & No & 4 & \\
\hline
\end{tabular}

En cuanto a los docentes que tienen el grado de Maestría (Tabla 13) que estuvieron capacitados y que tienen un espacio en casa para labores académicas $(\mathrm{N}=32)$, se obtuvo que $24(75 \%)$ realizan 
trabajo a distancia con sus estudiantes. Si se realiza un corte con 200 estudiantes o menos $(\mathrm{N}=28)$, el trabajo a distancia sube ligeramente al 78.6\%. Si solamente consideramos a los maestros con capacitación, sala de estudio y que tienen más de 200 alumnos $(\mathrm{N}=4)$ el trabajo a distancia cae al $50 \%$. En el lado opuesto; grado de Maestría, sin capacitación y sin lugar exclusivo en casa para trabajar (N=26), 14 maestros (53.8\%) están trabajando en línea y 12 (46.2\%) no lo están.

Tabla 13

Contraste de Características que Pueden Determinar el Trabajo a Distancia en Docentes con Grado de Maestría (N=107)

\begin{tabular}{|c|c|c|c|c|}
\hline Capacitación & Sala de Estudio & $\begin{array}{l}\text { Trabaja a } \\
\text { Distancia }\end{array}$ & $\begin{array}{c}\text { Menos de } 200 \\
\text { Estudiantes }\end{array}$ & $\begin{array}{l}\text { Más de } 200 \\
\text { Estudiantes }\end{array}$ \\
\hline \multirow[t]{4}{*}{ Sí (45) } & Sí (32) & Sí (24) & 22 & 2 \\
\hline & & No $(8)$ & 6 & 2 \\
\hline & No (13) & Sí (9) & 7 & 2 \\
\hline & & No (4) & 4 & \\
\hline \multirow[t]{4}{*}{ No (62) } & Sí (36) & Sí (11) & 9 & 2 \\
\hline & & No $(25)$ & 19 & 6 \\
\hline & No (26) & Sí (14) & 11 & 3 \\
\hline & & No (12) & 9 & 3 \\
\hline
\end{tabular}

Dentro del grado de Licenciatura (Tabla 14), con capacitación y espacio específico para trabajar $(\mathrm{N}=9), 6$ docentes $(66.7 \%)$ están trabajando a distancia. Al igual que en los casos anteriores: docentes con capacitación, sala de estudio y realizando el corte con menos de $200(\mathrm{~N}=7)$, son 6 (85.7\%) trabajando en línea. Si consideramos aquellos con capacitación, sala de estudio y más de 200 alumnos $(\mathrm{N}=2)$, el 100\% no trabaja a distancia. En contraparte; sin capacitación, ni sala de estudio ( $\mathrm{N}=13), 7$ maestros $(53.8 \%)$ estuvieron realizando trabajos en línea. 
Tabla 14

Contraste de Características que Pueden Determinar el Trabajo a Distancia en Docentes con Grado de Licenciatura $(N=41)$

\begin{tabular}{|c|c|c|c|c|}
\hline Capacitación & Sala de Estudio & $\begin{array}{l}\text { Trabaja a } \\
\text { Distancia }\end{array}$ & $\begin{array}{c}\text { Menos de } 200 \\
\text { Estudiantes }\end{array}$ & $\begin{array}{l}\text { Más de } 200 \\
\text { Estudiantes }\end{array}$ \\
\hline \multirow{4}{*}{ Sí (15) } & Sí (9) & Sí (6) & 6 & \\
\hline & & No (3) & 1 & 2 \\
\hline & No (6) & Sí (5) & 4 & 1 \\
\hline & & No (1) & 1 & \\
\hline \multirow[t]{4}{*}{ No (26) } & Sí (13) & Sí (5) & 5 & \\
\hline & & No (8) & 7 & 1 \\
\hline & No (13) & Sí (7) & 7 & \\
\hline & & No $(6)$ & 6 & \\
\hline
\end{tabular}

En la Figura 7 se hace una comparación entre docentes de diferente grado académico con capacitación y sala de estudio que están trabajando a distancia. La Figura 8 caracteriza a los maestros con capacitación, sala de estudio y separando a aquellos que tienen menos de 200 o más de 200 estudiantes. La Figura 9 se hace una comparación de los docentes que están trabajando a distancia, que no cuentan con capacitación ni sala de estudio en casa, en relación con el grado académico. 


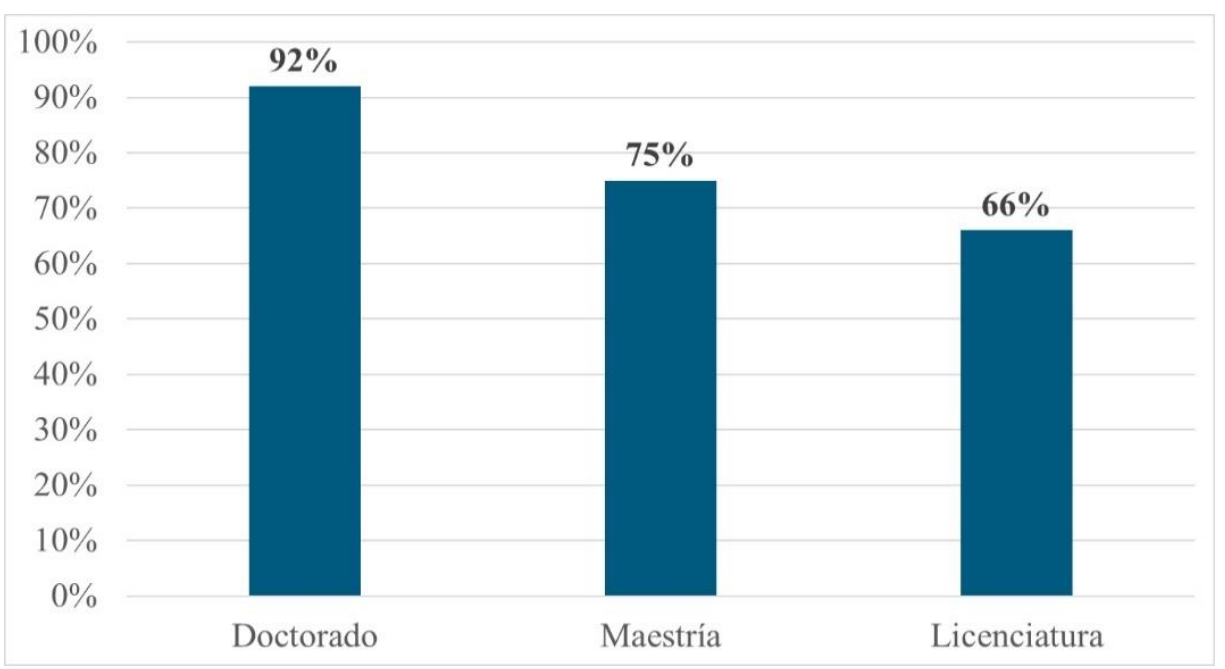

Figura 7. Docentes trabajando a distancia que cuentan con capacitación y sala de estudio en casa.

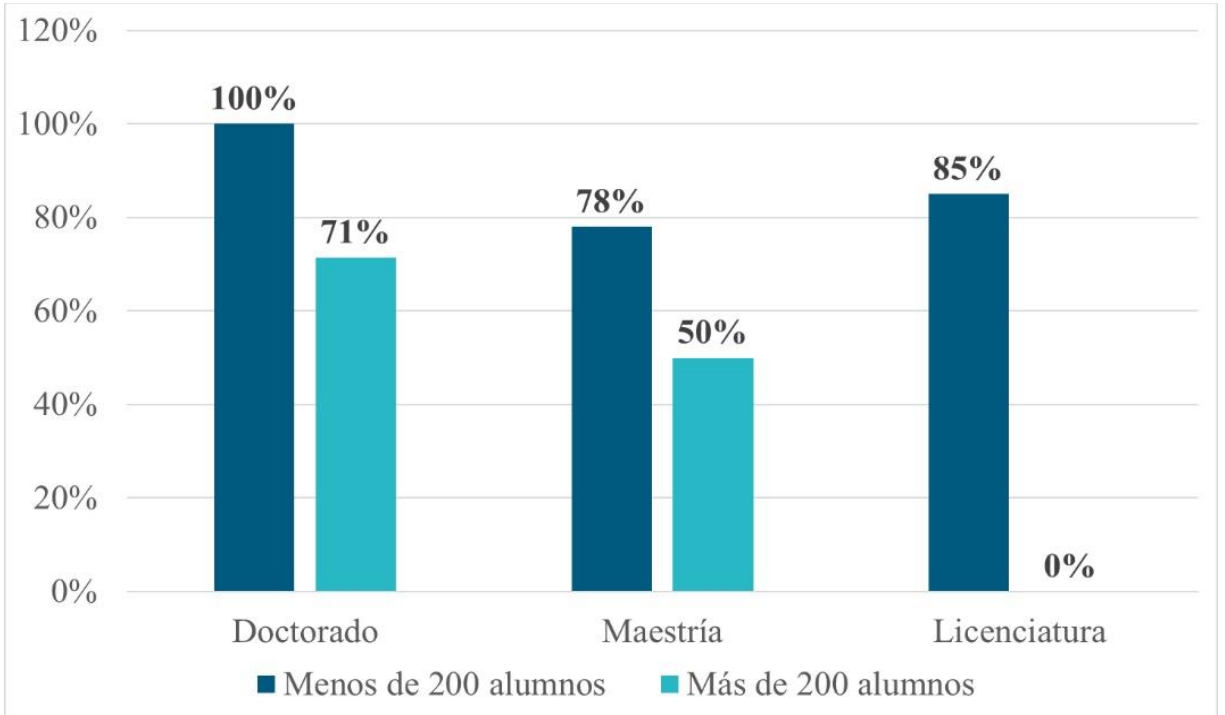

Figura 8. Docentes con capacitación y sala de estudio, en relación con su grado académico y número de estudiantes. 


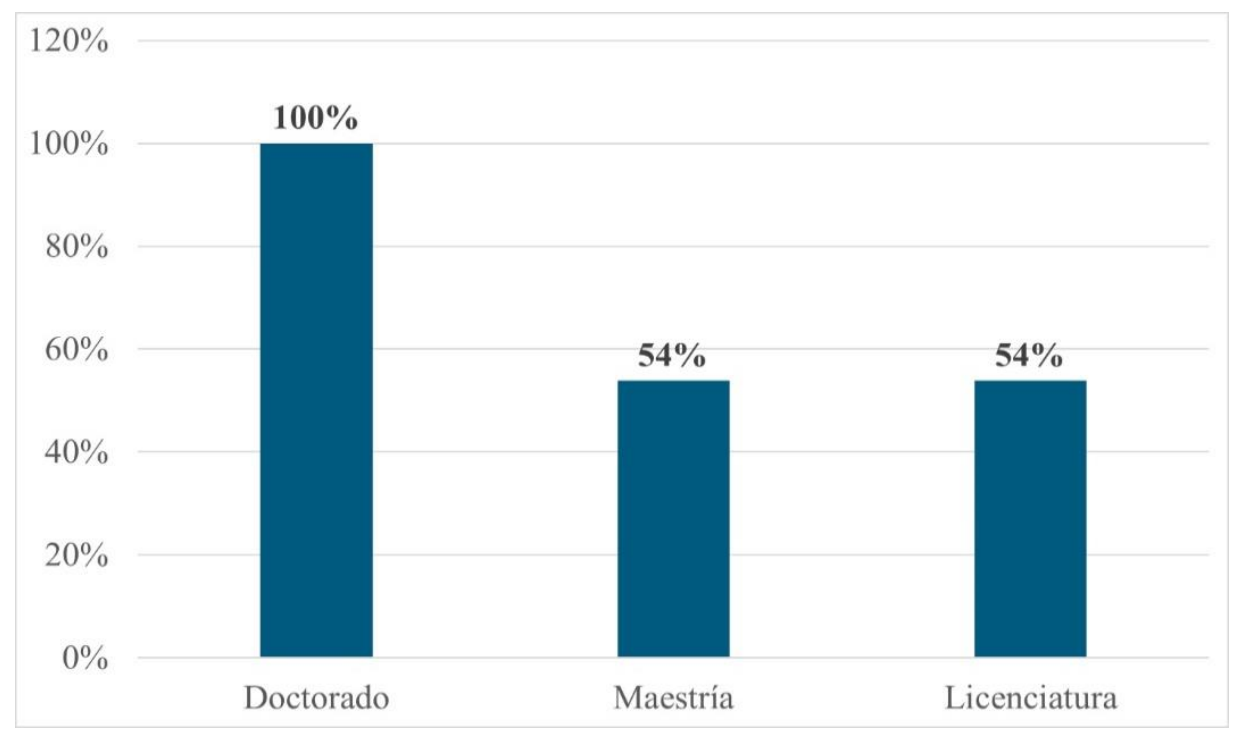

Figura 9. Docentes trabajando en línea que no cuentan con capacitación ni sala de estudio.

En el apartado emocional se les preguntó si habían experimentado alguna de estas situaciones durante la cuarentena: estrés, ansiedad, depresión, violencia doméstica, dinámica familiar complicada, enfermedad física, dificultades económicas, con opción de elegir más de una respuesta y la opción de poner alguna que no estuviera considerada (Tabla 15). La respuesta más seleccionada fue ansiedad por 132 docentes (64.7\%), seguida de estrés con 126 elecciones (61.8\%). La distinción por género la podemos observar en la Figura 10.

Tabla 15

Emociones Experimentadas por los Docentes Durante la Contingencia Sanitaria

\begin{tabular}{lccc}
\hline Emoción & Mujer & Hombre & General \\
\hline Ansiedad & 80 & 52 & 132 \\
Estrés & 86 & 40 & 126 \\
Depresión & 36 & 15 & 51 \\
$\begin{array}{l}\text { Dinámica familiar } \\
\text { complicada }\end{array}$ & 27 & 13 & 40 \\
$\begin{array}{l}\text { Dificultades } \\
\text { económicas }\end{array}$ & 22 & 11 & 33 \\
Enfermedad física & 9 & 8 & 17 \\
Violencia doméstica & 1 & 1 & 2 \\
\hline
\end{tabular}




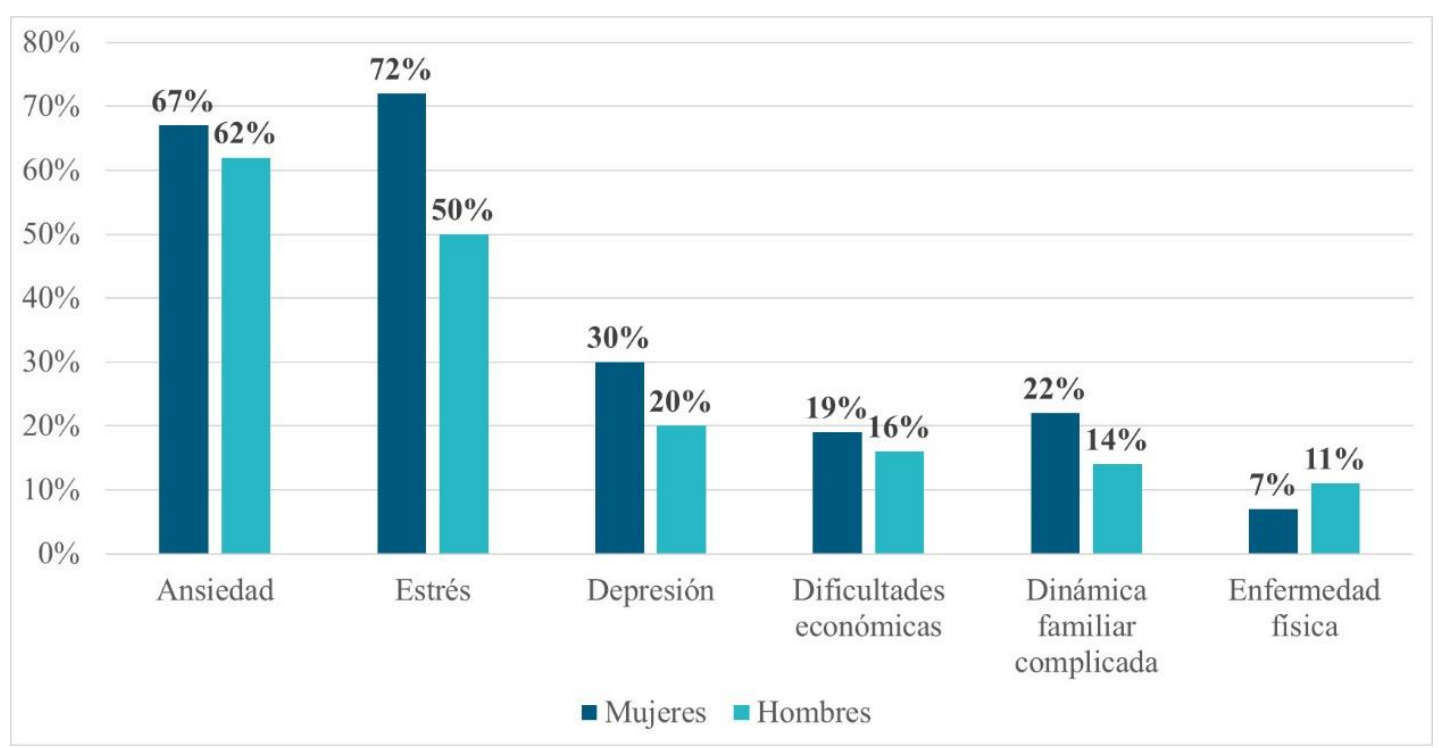

Figura 10. Problemas emocionales presentados en los docentes durante la contingencia.

Para conocer la percepción de los docentes acerca de la calidad de la enseñanza a distancia se les preguntó: ¿Consideras que la educación que se imparte a distancia puede ser de la misma calidad y obtener los mismos resultados que de forma presencial? (Tabla 16) 70 maestros (34.3\%) respondieron que sí puede ser de la misma calidad, mientras que 134 (65.7\%) estiman que no es posible que sea igual. Si se separa entre aquellos docentes que trabajan o no a distancia, se obtienen los siguientes resultados: de los que trabajan a distancia ( $\mathrm{N}=129), 56(43.4 \%)$ opinan que la calidad puede ser la misma. De los que no trabajan en línea (N=75), sólo 14 de ellos (18.7\%) opinan que la calidad puede ser igual al impartir clases a distancia.

\section{Tabla 16}

Percepción de los Docentes a la Calidad de la Educación a Distancia

\begin{tabular}{|c|c|c|}
\hline $\begin{array}{c}\text { Calidad de la Educación a } \\
\text { Distancia }\end{array}$ & Trabaja a Distancia & No Trabaja a Distancia \\
\hline Igual a la Presencial & 56 & 14 \\
\hline Menor a la Presencial & 73 & 61 \\
\hline Total & 129 & 75 \\
\hline
\end{tabular}


Para tratar de comprender el motivo de dicha creencia respecto a la calidad de la educación a distancia, se pidió a los docentes que argumentaran su respuesta a través de una pregunta abierta. Por parte de los que responden que la educación puede ser de la misma calidad, mencionan la importancia de la planeación, capacitación adecuada de todas las partes involucradas, la motivación que puede darles el uso de herramientas tecnológicas a los estudiantes:

- “Una buena programación de contenidos y actividades pueden lograr resultados comparables"

- "Si tiene objetivos claros, buena planeación y el trabajo suficiente detrás, para asegurar que se logren las competencias, creo que pueden tener los mismos resultados. En el caso de las asignaturas con prácticas de laboratorio definitivamente no lo creo posible."

- “Cuando se dominan las estrategias didácticas, las plataformas y tecnologías, se puede lograr lo mismo o incluso más, porque las nuevas generaciones se comunican mejor de esa manera y debemos ajustarnos a ellos".

De los que opinan que la calidad no es igual $(\mathrm{N}=75), 56$ docentes $(74.7 \%)$ hacen referencia a múltiples ventajas de una clase presencial, mencionan temas como la confianza que se puede dar entre docentes y estudiantes, una retroalimentación instantánea para corroborar que el objetivo de la clase se esté alcanzando o corregir al momento. Algunos vuelven a enfatizar en la falta de equipo, plataformas y conexión a internet de parte de todos los involucrados en este proceso. Se muestra incertidumbre sobre el aprendizaje real de los alumnos y la falta de interacción física es un punto preocupante para muchos docentes:

- "En la interacción y el contacto presencial existen puentes de comunicación inigualables"

- "Presencial puedes interactuar más fácilmente con los alumnos, puedes ver sus expresiones de duda para realizar las adecuaciones curriculares correspondientes, les dedicas tiempo de calidad a las clases y no das nada por hecho que te entendieron, asimismo los alumnos te demuestran sus emociones a través del 
contacto visual, sobre todo porque son pequeños y necesitan mucho la aprobación de su maestra."

- "Es muy complicado explicar cuando no tenemos una interacción cercana, viendo la expresión de si entendieron algo o no porque en el salón, siempre podía valorar si no me entendían, pero online no me doy cuenta de sus expresiones"

- "Tal vez se obtengan buenos resultados; sin embargo, no hay nada como el contacto humano, así, de frente, enseñándole al alumno a ser empático con sus semejantes. Enseñándole a convivir y trabajar en equipo, a aprender del otro y a aportar para todos."

- "El internet es sólo un medio, jamás sustituirá la clase presencial”

Para terminar la encuesta se les preguntó a los docentes que si no estuvieran en esta situación qué tipo de forma de trabajo elegirían, en la Tabla 17 se observan los resultados. 2 profesores (1\%) eligieron "que todas mis clases fueran en línea", 73 (35.8\%) respondieron "que todas mis clases fueran presenciales" por último, 129 (63.2\%) “que hubiera una combinación de ambas” (Figura11). Dentro de esta pregunta resalta el hecho de que 7 de cada 10 docentes que trabajan en línea $(\mathrm{N}=129)$ optarían porque fuera una combinación entre presencial y a distancia, y que más de la mitad de los que no están trabajando a distancia $(\mathrm{N}=75)$ eligieron el modelo combinado.

\section{Tabla 17}

Elección de Forma de Trabajar por Parte de los Docentes Fuera de la Contingencia

\begin{tabular}{lccc}
\hline \multicolumn{1}{c}{ Forma de Trabajo } & Trabaja a Distancia & $\begin{array}{c}\text { No Trabaja a } \\
\text { Distancia }\end{array}$ & General \\
\hline $\begin{array}{l}\text { Que todas mis clases } \\
\text { fueran en línea }\end{array}$ & 2 & & 2 \\
$\begin{array}{l}\text { Que todas mis clases } \\
\text { fueran presenciales }\end{array}$ & 39 & 34 & 73 \\
$\begin{array}{l}\text { Que hubiera una } \\
\text { combinación de ambas }\end{array}$ & 88 & 41 & 129 \\
Total & 129 & 75 & 204 \\
\hline
\end{tabular}


Finalmente, un hecho que resalta, es la preocupación por parte del maestro en relación con la falta de equipo, conexión a internet y en general a las condiciones socioeconómicas de sus estudiantes para poder desarrollar una educación a distancia adecuada (Tabla 11), sin lugar a duda, los comentarios en este sentido se presentaron en reiteradas ocasiones. Este suceso quizá se puede explicar por motivos como: la mayoría de los encuestados trabajan en el sector público (Tabla 10) y están conscientes de las carencias de sus estudiantes, ya que previo a la suspensión hubo un periodo de socialización entre ambas figuras. Otra razón puede ser que los maestros no son ajenos a las problemáticas sociales, culturales y económicas de nuestro país, incluidas las derivadas de la contingencia que estamos viviendo.

\section{Discusión}

Los resultados muestran una correlación entre la capacitación del docente (entendiéndola como la capacitación que recibe en su centro de trabajo, la formación continua que él mismo busca, su grado y formación académica) y la adopción de nuevos modelos de enseñanza que involucran a la tecnología (Tablas 2, 12, 13 y 14). Esto último ha repuntado recientemente en la enseñanza dentro del Nivel Superior, siendo el Blended Learning (combinación de herramientas digitales, recursos en línea, plataformas o EVEAs con clases presenciales) la modalidad más recurrente, sobre todo, por la ubicuidad que lo caracteriza. Lo anterior, aunado a las circunstancias de la pandemia, deja un vacío, sobre todo al momento de la integración de la estrategia y los recursos didácticos. Ese vacío ha provocado serias reflexiones al punto de llamarle Blended Teaching y no Blended Learning, debido al estado del conocimiento en el que se encuentra (Dziuban et al., 2018).

Lo anterior también explica por qué el grado académico es mayor entre los docentes que usaban la tecnología en sus clases previo a la contingencia, y por qué tuvieron la iniciativa de usarla como respuesta al cierre de escuelas (de la Tabla 9 se obtuvo que 9 de cada 10 docentes con doctorado están trabajando a distancia, mientras que el resultado obtenido con los maestros con nivel académico de maestría y licenciatura fue prácticamente el mismo (Tabla 9), 6 de cada 10 están trabajando a distancia).

Lo anterior está relacionado con el hecho de que el uso del BL no fue generalizado, sino mayormente era iniciativa de algunos docentes. Así pues, el BL no es una experiencia común entre 
los estudiantes, sobre todo, en el hecho de la supresión de las actividades y el trato presencial, volviendo aún más complejo afrontar para ellos el cierre de las instituciones educativas.

También se observa que el contar con menos o más de 200 estudiantes es un factor a considerar para trabajar o no a distancia, los docentes con menos de este número de alumnos tuvieron mayor predisposición a trabajar en línea, por el contrario, más de 200 alumnos demostró, dentro de los tres grados académicos (Tablas 12, 13 y 14), un descenso en el número de maestros trabajando a distancia. De acuerdo con la opinión de algunos docentes hay materias que, hasta el momento, no han encontrado la forma de trabajarlas en línea (Tabla 11), dado que su naturaleza es meramente práctica e incluso, requieren de equipos y espacios específicos e interacción física, entre otros.

Lo anterior es consistente con otros trabajos que consideran que el cambio de las interacciones presenciales por aquellas que ocurren por internet para fines educativos, deben ser idealmente graduales (Alexander et al., 2019) para tratar de usar las mismas reglas de interacción presenciales y hacer una posible adaptación de las mismas ( $\mathrm{Ng}, 2012$; Poulova et al., 2015), esto, como es sabido, no ocurrió debido a la magnitud de la emergencia. La situación es agravada por la falta de espacios para trabajar adecuadamente (Tablas 12, 13 y 14) y desde luego la combinación de roles como docentes, madres y padres de familia, que podemos observar en las citas de las docentes del grupo 4 (Tabla 11) en el apartado de resultados.

El modelo bola de nieve fue una forma de llegar a más docentes y una manera efectiva de encuestar durante el confinamiento, sin comprometer la salud de los informantes, no obstante, este estudio tiene como limitantes aquellas inherentes al modelo, como son: no controlar cómo se recaba la muestra y el tamaño final de ésta, por esa razón no se garantiza que los resultados sean representativos. En este sentido, el estudio recabó encuestas de 20 estados de la República Mexicana, pero estos datos no se pueden tomar como representativos para cada uno de ellos, ya que la mayoría pertenecen al estado de Zacatecas (66.2\%). Lo mismo ocurre con el nivel en que los docentes imparten clase, si bien se logró recabar información desde nivel primaria hasta doctorado, la mayoría pertenece a Nivel Medio Superior y Superior (Tabla 4). 


\section{Conclusiones}

Es necesario recalcar que este estudio se realizó en las primeras semanas de la contingencia, revelando las posibilidades, capacitación y recursos con los que contaban los profesores para dar una respuesta inicial sin apoyo institucional o como parte de un programa nacional.

Si bien el género no determina el trabajo a distancia, dentro del grupo de maestros que eligieron la falta de tiempo como problema para realizar dicha actividad, la mayoría de los que seleccionaron esta opción, fueron mujeres. Las respuestas abiertas analizadas indican la dificultad de empatar los múltiples roles personales con el profesional. Un aspecto que pudo ayudar a comprender más a fondo las dificultades de los docentes y que no se consideró en este estudio, fueron las condiciones socioeconómicas y familiares.

Tomando en cuenta la caracterización de los docentes que se hizo en este estudio, esto es, número de alumnos que atiende, grado de académico y trabajo previo con modelos mixtos. Se observa que aquellos docentes con menor número de alumnos y mayor grado de estudio y que trabajaban con modelos como el Blended Learning ofrecieron una respuesta inicial más eficaz y con menor grado de ansiedad, además, consideran la posibilidad de seguir usando los recursos en un futuro. En contraparte, los docentes que no tenían esas condiciones se mostraron más resistentes al cambio.

La mayoría de los encuestados perciben que la educación a distancia no puede alcanzar resultados de la misma calidad que la educación presencial, este hecho se acentúa en aquellos que no habían trabajado en esta modalidad. La calidad es subjetiva e influyen múltiples factores tales como: nivel educativo, número de estudiantes, equipo y conexión disponible, entre otros, es un hecho que el no haber impartido clases en línea limita la percepción de la eficacia que puede lograrse trabajando de esta forma.

También se puede considerar que el modelo BL tiene la ventaja de facilitar el cambio a un ambiente completamente en línea, que es lo que demanda la emergencia sanitaria. Para aquellos profesores que ya trabajaban de manera mixta: presencia y a distancia, fue un tránsito más sencillo, al final la experiencia, aunque forzada por la situación, puede resultar enriquecedora a la práctica docente. 
Queda como una prospectiva el analizar las impresiones de los alumnos y docentes sobre la vivencia de la pandemia, en términos educativos y, sobre todo, si el uso de la tecnología se volverá norma después de ella o se abandonará para volver a los modelos tradicionales.

Una contingencia mundial no es el panorama idóneo para buscar nuevas formas de trabajo, en el docente recayó una gran responsabilidad al tener que continuar con el ciclo escolar a distancia y al mismo tiempo enfrentar las distintas problemáticas sociales, laborales y personales. Aún no se vislumbra el regreso a las aulas, quizá cambie para siempre la forma habitual de enseñar, esto sin duda genera incertidumbre y abre un sinfín de dudas, pero también de posibilidades. Queda obligada la reflexión en cada docente: cómo adaptará su forma de trabajo y el rol que desempeñará en esta nueva realidad.

\section{Referencias}

Alcalá, M. G. (2019). Desigualdad en el acceso a internet en México y la afectación en el ejercicio del derecho humano a la información - Inequality in Internet access in Mexico and affecting the exercise of the human right to information. Nuevo Derecho, 15(24), 55-70. https://doi.org/10.25057/2500672X.1122

Alexander, B., Ashford-Rowe, K., Barajas-Murphy, N., Dobbin, G., Knott, J., McCormack, M., Pomerantz, J., Seilhamer, R., \& Weber, N. (2019). EDUCAUSE Horizon Report: 2019 Higher Education Edition.

Bartolomé, A., García-Ruiz, R., \& Aguaded, I. (2017). Blended learning: panorama y perspectivas. RIED. Revista Iberoamericana de Educación a Distancia, 21(1), 33. https://doi.org/10.5944/ried.21.1.18842

Crawford, J., Butler-Henderson, K., Rodolph, J., Malkawi, B., Glowatz, M., Burton, R., Magni, P., \& Lam, S. (2020). COVID-19 : 20 countries' higher education intra-period digital pedagogy responses. Journal of Applied Learning \& Teaching, 3(1), 1-20.

Dziuban, C., Graham, C. R., Moskal, P. D., Norberg, A., \& Sicilia, N. (2018). Blended learning: the new normal and emerging technologies. International Journal of Educational Technology in Higher Education, 15(1), 3. https://doi.org/10.1186/s41239-017-0087-5

Han, F., \& Ellis, R. A. (2019). Identifying consistent patterns of quality learning discussions in 
blended learning. Internet and Higher Education, 40, 12-19. https://doi.org/10.1016/j.iheduc.2018.09.002

Martínez Domínguez, M. (2018). Acceso y uso de tecnologías de la información y comunicación en México: factores determinantes. Paakat: Revista de Tecnología y Sociedad, 8(14), 1-18. https://doi.org/10.18381/Pk.a8n14.316

Mireles, A. (2020). El coronavirus pone a prueba el sistema educativo de México: más de la mitad de los estudiantes no tiene acceso a la educación en línea - Infobae. INFOBAE. https://www.infobae.com/america/mexico/2020/04/19/el-coronavirus-pone-a-prueba-el$\underline{\text { sistema-educativo-de-mexico-mas-de-la-mitad-de-los-estudiantes-no-tiene-acceso-a-la- }}$ educacion-en-linea/

$\mathrm{Ng}, \mathrm{W}$. (2012). Can we teach digital natives digital literacy? Computers and Education, 59(3), 1065-1078. https://doi.org/10.1016/j.compedu.2012.04.016

Owusu-Fordjour, C., Koomson, C. K., \& Hanson, D. (2020). The impact of Covid-19 on Learning. The perspective of Ghanaian Student. European Journal of Education Studies, 7(3), 88-101. https://doi.org/10.5281/zenodo.3753586

Poulova, P., Simonova, I., \& Manenova, M. (2015). Which One, or Another? Comparative Analysis of Selected LMS. Procedia - Social and Behavioral Sciences, 186, 1302-1308. https://doi.org/10.1016/j.sbspro.2015.04.052

Salinas Ibáñez, J., Benito Crosetti, B. de, Pérez Garcias, A., \& Gisbert Cervera, M. (2018). Blended Learning, más allá de la clase presencial. RIED. Revista Iberoamericana de Educación a Distancia, 21(1), 195-213.

Sánchez Mendiola, M., Martínez Hernández, A. M., Torres Carrasco, R., de Agüero Servín, M., Hernández Romo, A. K., Benavides Lara, M. A., Rendón Cazales, V., \& Jaimes Vergara, C. (2020). Retos educativos durante la pandemia de COVID-19: una encuesta a profesores de la UNAM. Revista Digital Universitaria, 21(3), 1-24. https://doi.org/10.22201/codeic.16076079e.2020.v21n3.a12

UNESCO. (2020). School closures caused by Coronavirus (Covid-19). https://en.unesco.org/covid19/educationresponse

Veiga, J., Cabo, D. E., De, E., Fuente Díez, L. A., \& Zimmermann Verdejo, M. (2008). Modelos de Estudios en Investigación Aplicada: Conceptos y criterios para el diseño. Med Segur $\operatorname{Trab}$ (Vol. 210) 